\title{
Assessing Rough-and-tumble Play Behavior in Juvenile Rats
}

Jonathan W. VanRyzin ${ }^{1, *}$, Ashley E. Marquardt ${ }^{2}$ and Margaret M. McCarthy ${ }^{1,2, *}$

${ }^{1}$ Department of Pharmacology, University of Maryland School of Medicine, Baltimore, Maryland, United States of America; ${ }^{2}$ Program in Neuroscience, University of Maryland School of Medicine, Baltimore, Maryland, United States of America

*For correspondence: jonathan.vanryzin@som.umaryland.edu; mmccarthy@som.umaryland.edu

\begin{abstract}
[Abstract] Play is a complex social behavior that is highly conserved across mammals. In most species, males engage in more frequent and vigorous play as juveniles than females, which reflects subtle yet impactful sex differences in brain circuitry and development. In this protocol, we describe a behavioral testing paradigm to assess social play in male and female juvenile rats. We highlight the behavior scoring criteria for distinguishing rough-and-tumble play from other play-related social behaviors. By analyzing both sexes, play behavior can be leveraged as a powerful tool to understand the sex-specific development and expression of social behavior.
\end{abstract}

Keywords: Social play, Sex differences, Behavior, Brain development, Rats, Social behavior, Play fighting, Rough-and-tumble play

[Background] Play is a highly conserved social behavior expressed by juveniles in several mammalian species, from rodents to humans (Auger and Olesen, 2009). Play is highly complex, involving dynamic interactions between conspecifics and is necessary for the development of appropriate social, cognitive, and affective behaviors later in life (Van den Berg et al., 1999; Von Frijtag et al., 2002; Baarendse et al., 2013). In many species, males engage in more frequent and vigorous play, called rough-and-tumble play or play fighting. This sex difference in behavior is developmentally programmed and is the result of sexual differentiation of the brain (Auger and Olesen, 2009; Thornton et al., 2009).

Here we describe a protocol to assess juvenile play using male and female rats. Each day throughout the juvenile period, rats are paired with an age-, sex-, and treatment-matched partner and play behavior is analyzed. In this way, this protocol is used to assess sex differences in baseline or "naturalistic" play. When combined with early-life pharmacological or genetic manipulation, this protocol can be used to study the processes of brain sexual differentiation and the development of social behavior circuitry (Vanderschuren et al., 2016; VanRyzin et al., 2019). Alternatively, the protocol can be performed as a single-trial to study sex differences in the activation of neural circuitry underlying play (Veenema et al., 2013; Bredewold et al., 2018). 


\section{Materials and Reagents}

1. Male and female juvenile (4-5-week-old) Sprague-Dawley rats Notes:

a. We breed and maintain our own colony at the University of Maryland School of Medicine. This facilitates early-life treatments with pharmacological agents predicted to affect masculinization and avoids stress due to animal shipping or relocation. Animals are kept on a 12:12 $\mathrm{h}$ reverse light:dark cycle.

b. Rats are tested as sex-, treatment- and age-matched non-sibling pairs. These "play pairs" will remain constant across multiple days of testing such that each animal is playing with the same play partner every day. To achieve this, we plan experiments to include rats from at least two separate litters. Depending on the number of experimental conditions, we will test at least two cohorts of play pairs (2 sets of paired litters, or 4 litters total) (see Figure $1 A$ for experimental design and timeline).

c. At birth, litter size is culled to 12-14 pups per dam to avoid confounds of over/under nutrition and maternal care. Litters are kept as sex- and treatment-balanced as possible, and litters with an initial sex ratio of > 2:1 males:females (or vice versa) are not used in our experiments due to potential in utero effects of prenatal testosterone.

2. Pharmacological agents of interest

Note: Depending on the hypothesis under study and the experimental timing of treatments, animals may need to be treated much earlier in life (prenatal exposure, postnatal masculinization, etc.; see VanRyzin et al., 2019 for examples).

A

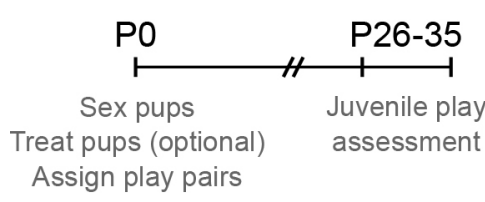

Dam $1 \longrightarrow$ FFFFFF M M M M M M $\uparrow \uparrow \uparrow \uparrow \uparrow \uparrow \uparrow \uparrow \uparrow \uparrow \uparrow \uparrow$
B
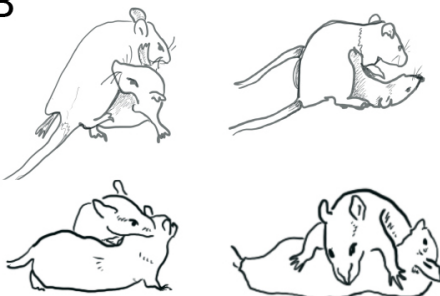

PIN
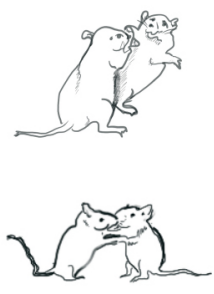

Box

Figure 1. Representative experimental timeline and behavior illustrations. A. On the day of birth, pups from two litters are sexed, culled and treated (if necessary), and assigned to sexand treatment-matched pairs with the opposite litter. The pups are allowed to grow undisturbed until the juvenile age. Beginning at P26, play behavior can be assessed daily throughout the juvenile period. B. Illustrations depicting rats engaging in the three main rough-and-tumble play behaviors-pouncing, pinning, and boxing. 


\section{Equipment}

1. Custom plexiglass testing arena $49 \mathrm{~cm} \times 37 \mathrm{~cm}, 24 \mathrm{~cm}$ high (see Figure $2 \mathrm{~A}$ )

2. Behavioral testing room equipped with red light

3. One to two lamp stands with red light bulbs for illumination of the testing arenas

4. Video recording device and tripod stand

Note: We typically use a smart phone with a tripod adapter to record behavioral tests.

5. TEK-Fresh bedding (Envigo, catalog number: 7099)

6. Fur-marking pen or permanent marker (Stoelting, catalog number: 50440-1)

7. Stopwatch or timer

8. Cleaning solution

Note: Several different cleaning solutions may be used to sanitize the arenas after testing. We typically use MB-10 (Quip Laboratories).

\section{Procedure}

1. Prior to the first test day, set up the behavioral testing room.

a. Ensure that the red light illumination provides appropriate contrast for video recording.

b. If testing more than one pair of animals at a time, arrange all testing arenas such that they can be adequately recorded by the available recording equipment. Using a smart phone camera, we are able to record 4 arenas simultaneously with sufficient detail and resolution. See Figure 2B.

A

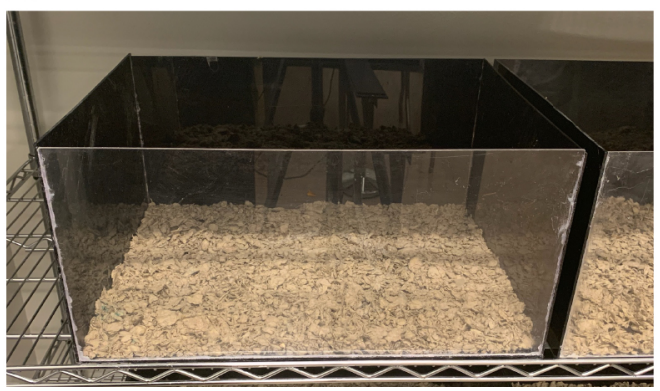

B

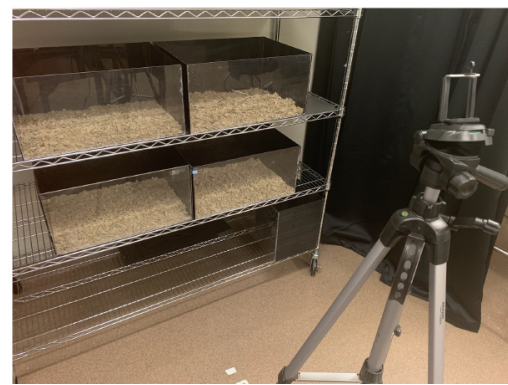

Figure 2. Testing arena and set up. A. Image of a single behavior testing arena. B. Image of four testing arenas arranged in front of a tripod for video recording.

c. Fill each testing arena with enough TEK-fresh bedding to cover the bottom of the arena (approximately 2-3 cm deep).

d. Plan play pairs and the order of behavioral testing. The order of testing needs to be changed each day to counterbalance the time of testing for each group across all test days.

2. On the day of testing, move the animals (in their home cage) from the colony room into the behavioral testing room to allow them to habituate to the experimental environment (approx. 20 
min prior to testing and at least $1 \mathrm{~h}$ after the start of the dark cycle). Testing during the dark phase (the active phase for the rat) is essential for proper behavioral analysis.

3. Mark one of the animals in each play pair either with a fur-marking pen (on the head or back) or by striping the tail with a permanent marker. This facilitates behavioral analysis as it is easier to attribute specific behaviors to the marked or unmarked animal.

4. Once the animals have habituated, place an animal pair into the testing arena and allow the animals to acclimate to the arena for $2 \mathrm{~min}$.

5. After acclimation, record behavior for $10 \mathrm{~min}$.

6. Once the test has concluded, stop the recording. Remove the animals from the testing arena and place them back in their home cage.

7. Repeat Steps 4-6 as many times as needed until all animals have been tested.

8. On subsequent days, repeat Steps 2-7. We typically perform the test for 4 continuous days (P27-30) to capture the peak sex difference in play behavior; however, the test can be repeated each day throughout the entire juvenile period (approximately P26-35).

Notes:

a. During the test, the experimenter should remain behind a curtain or out of sight of the testing arenas. Additionally, maintain quiet in the room as external noises may disrupt behavior.

$b$. It is not necessary to clean the testing arena or change the bedding between trials. At the conclusion of each test day, the arena should be cleaned (using a cleaner of the experimenter's choosing) and bedding replaced in preparation for the next day.

c. Be sure to keep the red light intensity to a minimum to avoid creating an artificially stressful testing environment. The lighting for our behavior testing is typically $<50$ lux. Additionally, red light should be used for Sprague Dawley rats as they are albino and their inability to see red light keeps the testing environment as close to a natural dark phase as possible for optimal behavior testing.

d. While rats do emit ultrasonic vocalizations during play, this does not impact the expression of play (Kisko et al., 2015), and does not confound testing more than 1 pair of animals at a time.

\section{Data analysis}

1. Behavior is scored offline by an experimenter blind to the sex and treatment of the animals. Note: Scoring may be done by hand or by using keylogger software.

2. The animal performing a given behavior receives a score for that behavior. Thus, one play pair yields two data points. This helps improve interpretability of the data, as high- and low-playing animals will not be averaged with their play partner's behavior. Moreover, scoring behavior for each animal allows for an analysis of each individual animal over time to get an average play value across all test days.

3. To assess rough-and-tumble play behavior, we score the following behaviors (see Videos 1-3 and Figure 1B): 
a. Pouncing-Scored when one animal launches itself toward the other's nape or back. This is often a vigorous motion and is scored to the animal performing the pounce.

Note: Animals may forcefully bump into each other or perform "low effort" pounces. These are generally not scored as the intent to initiate play is much more ambiguous.

b. Pinning-Scored when a pouncing animal flips the other completely onto its back (all four limbs off the ground). A pin is scored to the pouncing animal, and often will be scored directly after a pounce.

Note: Pinning is often the result of the animal that is being pounced on continuing the play bout by rolling onto their back in a defensive position. However, the animal may flip over onto its side, keeping weight on their feet. In these cases, a pin is not scored.

c. Boxing-Scored when both animals rear up on their hind legs and paw at each other. Both animals receive a score for boxing.

Note: Boxing is much less frequent than either pouncing or pinning and is observed often following bouts of chasing, when the animal being chased turns and confronts the chaser.

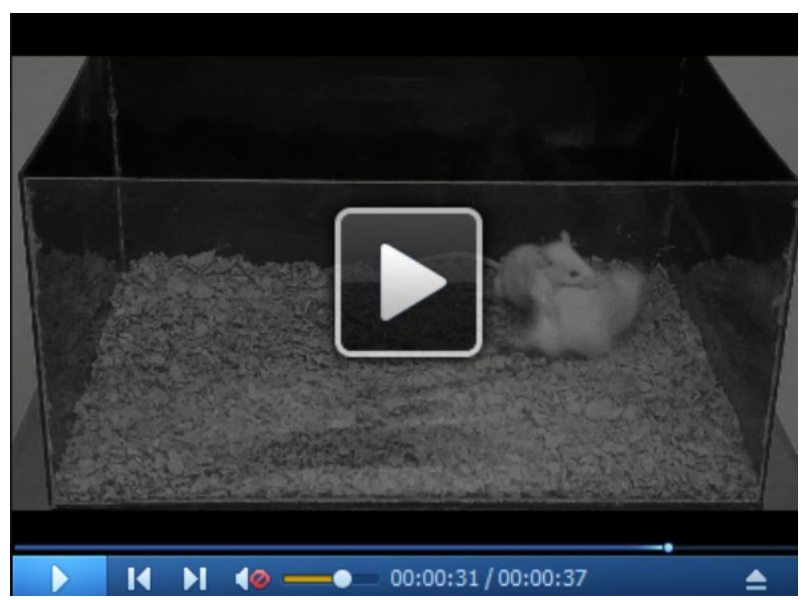

Video 1. Sample behavioral sequence featuring pouncing and pinning. (All animal procedures described here are performed in accordance with the Animal Care and Use Committee's regulations at the University of Maryland School of Medicine (Protocol \# 0517007 , 06/30/20).) 


\section{bĭ́-protocol

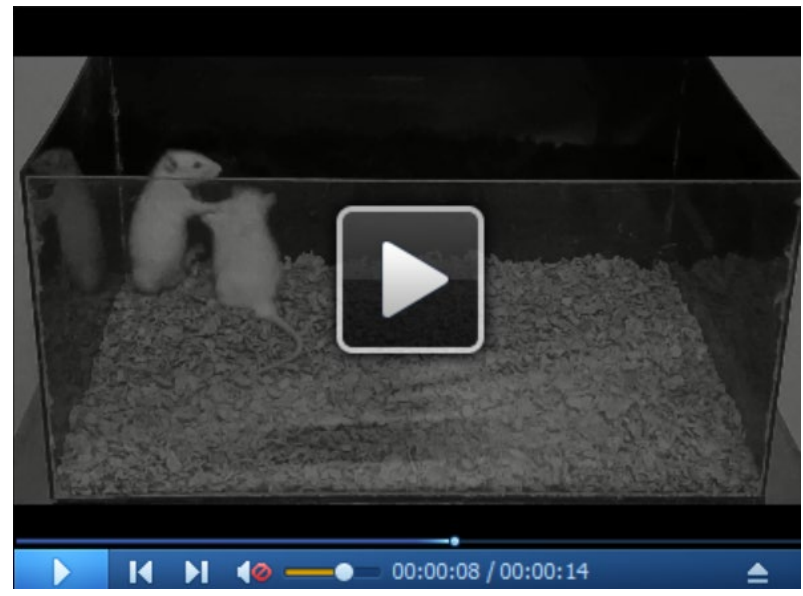

Video 2. Sample behavioral sequence featuring boxing. (All animal procedures described here are performed in accordance with the Animal Care and Use Committee's regulations at the University of Maryland School of Medicine (Protocol \# 0517007, 06/30/20).)

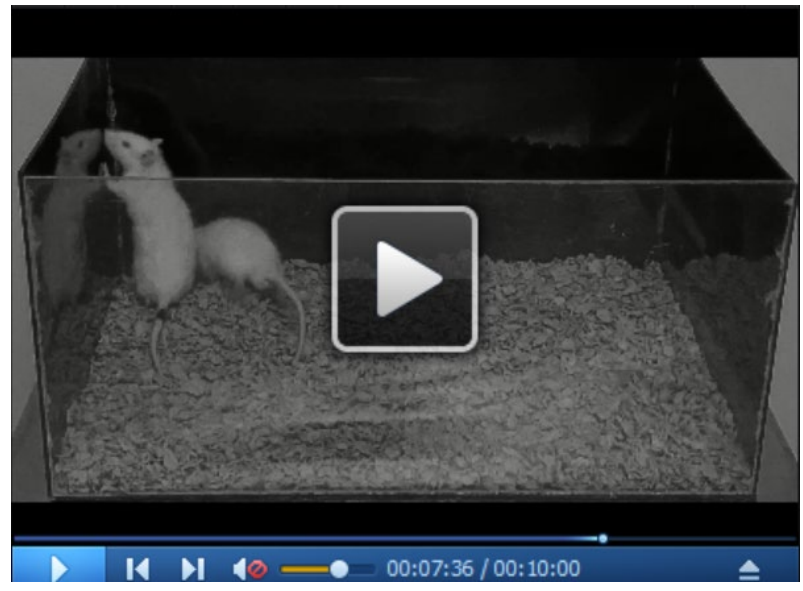

Video 3. Sample video of an entire play behavior test. (All animal procedures described here are performed in accordance with the Animal Care and Use Committee's regulations at the University of Maryland School of Medicine (Protocol \# 0517007, 06/30/20).)

4. The following behaviors can also be scored but are considered to be social or locomotor play behaviors:

a. Chasing Scored when one animal actively chases the other. Only the "chaser" receives a score for chasing.

Note: Typically, chasing precedes pounces or boxing behavior.

b. Bouncy gait-Scored when one animal moves around the arena in a series of "jumps" or "hops".

5. The following non-play social behaviors can also be scored:

a. Anogenital sniffing-Scored when one animal actively sniffs the anogenital region of the other.

b. Licking and grooming-Scored when one animal actively licks or grooms the head or anogenital region of the other. 
c. Huddling-Scored when both animals are sitting in very close proximity/touching each other but are not engaged in any other form of social behavior (sniffing/licking/grooming). Both animals receive a score for huddling.

d. Crawling over/under-Scored when one animal crawls over or under the other.

Note: This typically happens during periods of environmental exploration

6. The following environmental exploration behaviors can also be scored:

Note: These behaviors are best scored as a duration of time the animal spends engaged in each category.

a. Digging and burrowing in the bedding-Scored as the duration of time one animal burrows or nests in the bedding.

b. Chewing on the bedding-Scored as the duration of time one animal holds a piece of bedding in their paws and chews on it.

c. General arena investigation-Scored as the duration of time one animal spends sniffing or rearing up on their hind legs to explore the walls of the testing arena.

7. Statistical Analysis:

a. If age is an experimental factor of interest, the data may be represented as an average for each day and analyzed using a repeated-measures ANOVA (see Figure 3A and Argue and McCarthy, 2015 for more examples).

b. If age is not of interest, the data may be averaged across all days for an individual animal and analyzed using a $t$-test or ANOVA depending on the number of experimental groups (see Figures 3B-3E and VanRyzin et al., 2019 for more examples).

A

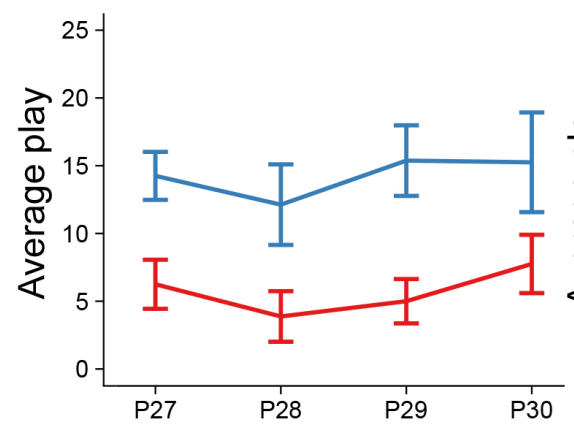

C

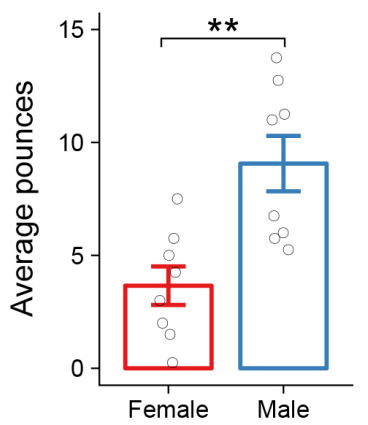

B

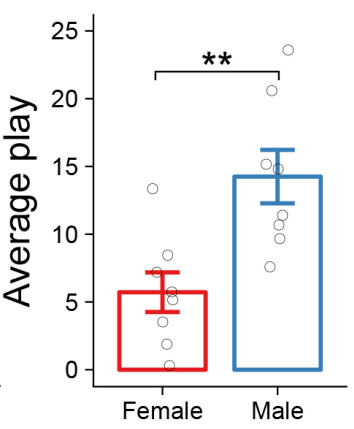

E

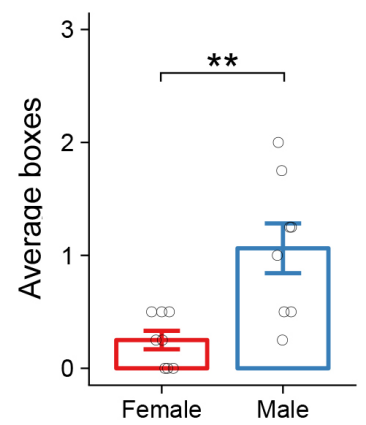

Figure 3. Sample data. A. Sample data showing total play (pounces, pins, and boxes) for males 
and females daily from P27-P30. B. Sample data showing the total play data from A as an average across all four testing days. C-E. Sample data from B broken down into pounces (C), pins $(D)$, and boxes $(E)$. Data are represented as the mean \pm SEM. Blue and red lines/bars indicate males and females, respectively. Open circles indicate values for individual animals. * $=P<0.05,{ }^{* *}=P<0.01$.

\section{$\underline{\text { Notes }}$}

1. Prior to the first test day, animals can be tested in an open field test for general locomotor activity and exploratory behavior to rule out any confounds of activity differences between groups. In general, we have never found baseline activity differences between males and females, but experimental manipulations may affect this.

2. Filling the testing arena with too much bedding can lead to a decrease in social behavior and an increase in environmental exploration, as the animals will begin to burrow under the bedding.

3. We test animals on a reverse light:dark cycle and begin behavioral testing 1-2 $\mathrm{h}$ after the onset of the dark cycle. It is important to keep the timing of behavioral analysis consistent across days to avoid any circadian influences on behavior.

4. Depending on the number of test days, age of the animals, and pharmacological manipulations, animals may begin to display sex behavior at the onset of puberty (approximately P40 in our colony). We do not score sex behavior as a social behavior in this paradigm, as it occurs very rarely in our experience; however, if it is occurring frequently, it can be analyzed as a separate set of behaviors to determine if puberty has occurred early.

5. Male and female play behavior is expressed along a continuum from high to low play. While males, on average, are on the "high" end of the continuum relative to females, there is a fair amount of natural individual variability. Each cohort of animals will likely have a few high-playing females and low-playing males. This natural behavioral variability is not a reason to exclude these animals from analysis; rather, plan experiments with a sufficient sample size to account for this. We recommend at least 8 animals per sex.

6. Play pairs should be same-sex, same-treatment. Mixing sexes or treatments within a given play pair may result in an intermediate play phenotype (i.e., a more playful partner will increase a low playing rats play and vice versa). See Argue and McCarthy, 2015 for an example.

\section{Acknowledgments}

This work was funded by the National Institutes of Health R01MH52716 and R01DA039062 to M.M.M.This protocol was adapted from Argue and McCarthy, 2015, and Vanryzin et al., 2019.

\section{Competing interests}


The authors have no competing interests to declare.

\section{Ethics}

All animal procedures described here are performed in accordance with the Animal Care and Use Committee's regulations at the University of Maryland School of Medicine (Protocol \# 0517007 , 06/30/20).

\section{References}

1. Argue, K. J. and McCarthy, M. M. (2015). Characterization of juvenile play in rats: importance of sex of self and sex of partner. Biol Sex Differ 6: 16.

2. Auger, A. P. and Olesen, K. M. (2009). Brain sex differences and the organisation of juvenile social play behaviour. J Neuroendocrinol 21(6): 519-525.

3. Baarendse, P. J. J., Counotte, D. S., O'Donnell, P. and Vanderschuren, L. J. M. J. (2013). Early social experience, is critical for the development of cognitive control and dopamine modulation of prefrontal cortex function. Neuropsychopharmacol 38:1485-1494.

4. Bredewold, R., Nascimento, N. F., Ro, G. S., Cieslewski, S. E., Reppucci, C. J., and Veenema, A. H. (2018). Involvement of dopamine, but not norepinephrine, in the sex-specific regulation of juvenile socially rewarding behavior by vasopressin. Neuropsychopharmacol 43:2109-2117.

5. Kisko, T. M., Euston, D. R. and Pellis SM. (2015). Are 50-khz calls used as play signals in the playful interactions of rats? III. The effects of devocalization on play with unfamiliar partners as juveniles and as adults. Behav Processes 113:113-121.

6. Thornton, J., Zehr, J. L. and Loose, M. D. (2009). Effects of prenatal androgens on rhesus monkeys: a model system to explore the organizational hypothesis in primates. Horm Behav 55(5): 633-645.

7. Vanderschuren, L. J., Achterberg, E. J. and Trezza, V. (2016). The neurobiology of social play and its rewarding value in rats. Neurosci Biobehav Rev 70: 86-105.

8. Van den Berg, C. L., Hol, T., Van Ree, J. M., Spruijt, B. M., Everts, H. and Koolhas, J. M. (1999). Play is indispensable for an adequate development of coping with social challenges in the rat. Dev Psychobio/ 34(2):129-138.

9. Vanryzin, J., Marquardt, A., Argue, K., Vecchiarelli, H. A., Ashton, S., Arambula, S. E., Hill, M. N. and McCarthy, M. M. (2019). Microglial phagocytosis of newborn cells is induced by endocannabinoids and sculpts sex differences in juvenile rat social play. Neuron 102(2):435449.

10. Veenema, A. H., Bredewold, R. and De Vries, G. J. (2013). Sex-specific modulation of juvenile social play by vasopressin. Psychoneuroendocrinology 38(11): 2554-2561. 
11. Von Frijtag, J. C., Schot, M., van den Bos, R. and Spruijt, B. M. (2002).Individual housing during the play period results in changed responses to and consequences of a psychosocial stress situation in rats. Dev Psychobiol 41(1): 58-69. 